\title{
Crystallization Kinetics of the Phase Change Material GeSb 6 Te Measured with Dynamic Transmission Electron Microscopy
}

\author{
M. M. Winseck, ${ }^{1}$ H.-Y. Cheng, ${ }^{2}$ G. H. Campbell, ${ }^{3}$ and M. K. Santala ${ }^{1}$ \\ 1. Department of Mechanical, Industrial, and Manufacturing Engineering, Oregon State University, \\ Corvallis, OR, USA \\ 2. IBM/Macronix PCRAM Joint Project, Macronix International Co., Ltd., Hsinchu, Taiwan \\ 3. Materials Science Division, Lawrence Livermore National Laboratory, Livermore, CA, 94550, USA
}

Phase change materials (PCMs) are a class of materials with distinct optical and electrical properties in the amorphous and crystalline phases that make them useful for optical information storage and resistivity-based solid-state memory devices [1]. For memory applications, it must be possible to switch between the amorphous and crystalline phases by rapid heating in just a few nanoseconds. The crystallization kinetics of PCMs are of primary importance as they directly impact device switching speed, but even basic quantities, such as crystal growth rates, are difficult to measure experimentally during highly-driven laser- or current-induced crystallization.

$\mathrm{GeSb}_{6} \mathrm{Te}$ is a chalcogenide-based PCM that has shown great potential for use in solid-state memory devices [2]. Only a small set of materials satisfy the requirements of adequate contrast in properties, amorphous stability at low temperatures, and rapid crystallization. There is a continuing search for materials that may be switched faster and that have a higher crystallization temperature, $\mathrm{T}_{\mathrm{x}}$, in order to make the speed of new PCM-based memory competitive against other types of storage and random access memory and to broaden the conditions under which PCMs may be used. GeSb ${ }_{6}$ Te has been shown to be a promising candidate for storage-class memory, because its $T_{x}$ is substantially higher than that of other commonly used PCMs, and because melt-quenched amorphous films have been shown to crystalize rapidly during laser crystallization [2]. The crystallization kinetics of other Sb-rich PCM alloys have been studied, but the $\mathrm{GeSb}_{6} \mathrm{Te}$ composition has only recently been considered as a candidate PCM material and its kinetics have not been extensively studied.

The crystallization kinetics of 30-nm amorphous thin films of $\mathrm{GeSb}_{6}$ Te were followed with dynamic transmission electron microscopy, a photo-emission electron microscopy technique with nanosecondscale time resolution. Crystallization was induced with a Nd:YAG laser (1064 nm wavelength, $135 \pm 5$ $\mu \mathrm{m} 1 / \mathrm{e}^{2}$ diameter, 12-ns FWHM duration) directed onto the specimen in the TEM column. Crystallization was induced with laser pulses ranging from $1.08-1.43 \mathrm{~mJ}$ and nine-frame movies of crystal growth were taken over a $4.6 \mu$ s period, e.g. Figure 1. The nucleation rate is observed to be very low. The growth rates are very high, up to $10.8 \mathrm{~m} / \mathrm{s}$ for amorphous as-deposited films (Figure 2), and even higher for an amorphous film subject to sub-threshold laser annealing before crystallization. The crystallization is reminiscent of explosive crystallization of elemental semiconductors both in the magnitude of the growth rate and in the resulting crystalline microstructures [3].

[1] M. Wuttig and N. Yamada, Nature Materials 6 (2007), p. 824.

[2] H.-Y. Cheng, S. Raoux, and L.J. Jordan-Sweet, Journal of Applied Physics 115 (2014), p. 5.

[3] This work performed, in part, under the auspices of the U.S. Department of Energy, Office of Basic Energy Sciences, Division of Materials Sciences and Engineering by Lawrence Livermore National Laboratory under Contract DE-AC52-07NA27344. 


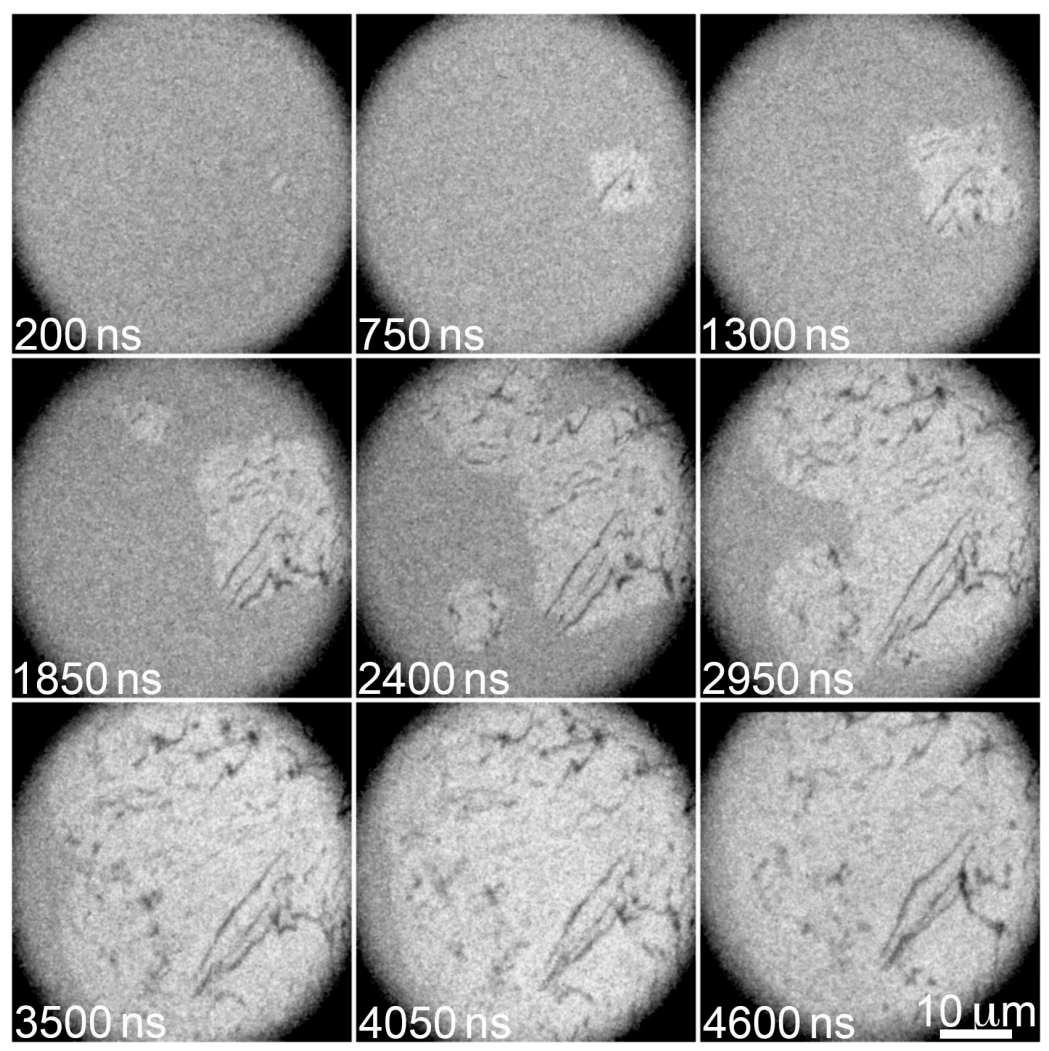

Figure 1. Bright field images showing the growth of crystalline grains in an amorphous $\mathrm{GeSb}_{6} \mathrm{Te}$ thin film in a nine-frame movie formed by nine 50-ns electron pulses induced by a $1.29 \mu \mathrm{J}$ laser pulse. The time in each frame is relative to the specimen laser intensity with an uncertainty of $\pm 3 \mathrm{~ns}$.

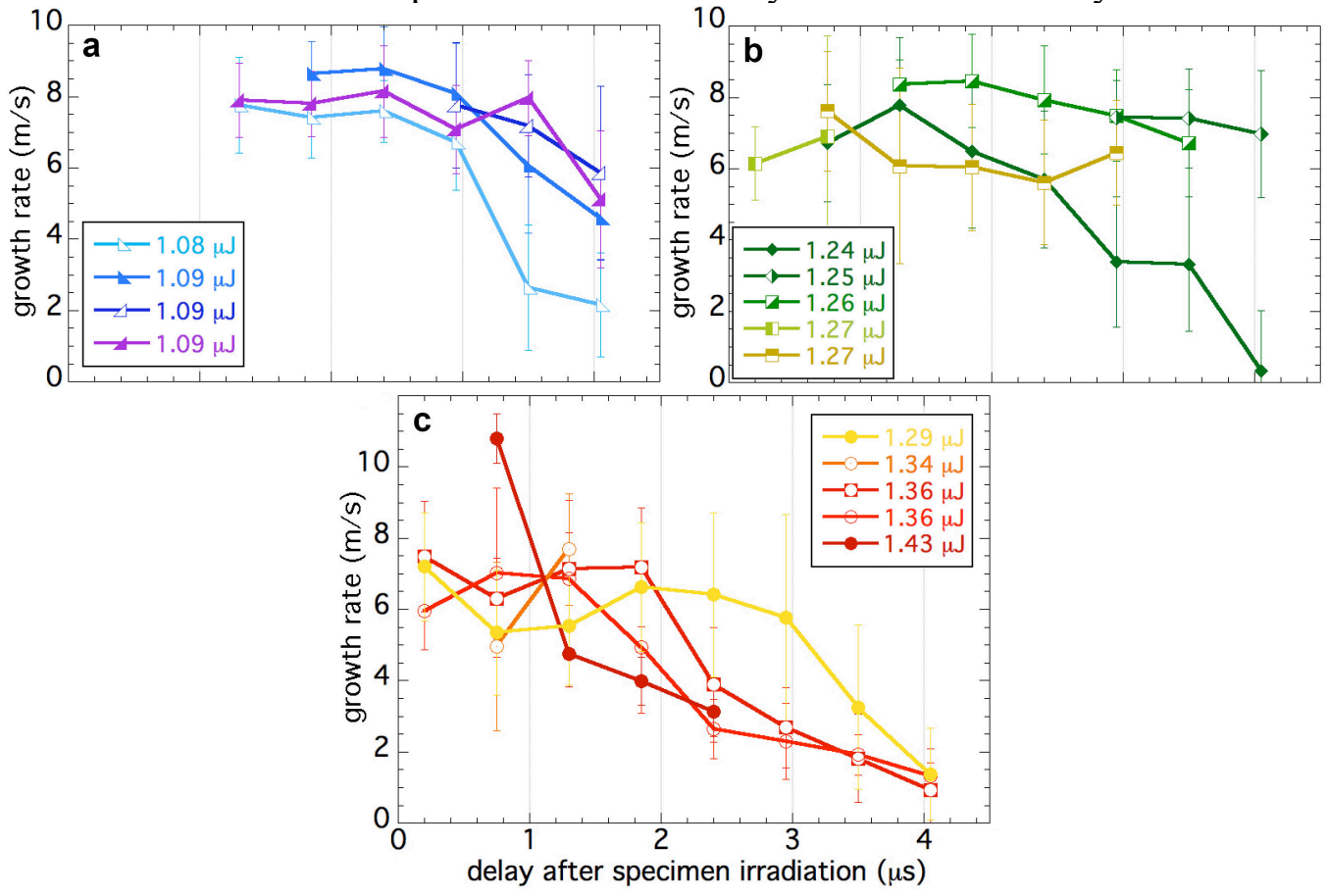

Figure 2. Crystal growth rates versus time since the specimen laser pulse. The first point for each specimen indicates the first frame with visible crystalline material. The growth rates are plotted for different laser energy ranges on separate plots for clarity. 